\title{
Asymptotic behavior of solutions to a class of coupled nonlinear parabolic systems
}

\author{
Yan Leng' ${ }^{1}$ Yuanyuan $\mathrm{Nie}^{1 *}$ (1) and Qian Zhou'
}

"Correspondence: nieyy@jlu.edu.cn ${ }^{1}$ School of Mathematics, Jilin

University, Changchun, China

\section{Springer}

\begin{abstract}
This paper studies the Cauchy problem to a class of coupled nonlinear parabolic systems and investigates the asymptotic behavior of solutions to the problem. The blow-up theorem of Fujita type is established by the integral estimation and suitable supersolutions. Moreover, the critical Fujita exponent determined by the diffusion and the spatial dimension is given.
\end{abstract}

MSC: 35B33; 35K20; $35 \mathrm{~K} 58$

Keywords: Critical Fujita exponent; Asymptotic behavior; Blow-up theorem

\section{Introduction}

We investigate the asymptotic behavior of solutions to the Cauchy problem of the coupled nonlinear parabolic systems by the following initial value problem:

$$
\begin{aligned}
& \frac{\partial u}{\partial t}=\Delta u^{m}+v^{p}, \quad x \in \mathbb{R}^{n}, t>0, \\
& \frac{\partial v}{\partial t}=\Delta v^{m}+(|x|+1)^{2(q-p) /(p-m)} u^{q}, \quad x \in \mathbb{R}^{n}, t>0, \\
& u(x, 0)=u_{0}(x), \quad v(x, 0)=v_{0}(x), \quad x \in \mathbb{R}^{n},
\end{aligned}
$$

where $q \geq p>m>1$ and $0 \leq u_{0}, v_{0} \in C_{0}\left(\mathbb{R}^{n}\right)$ are nontrivial.

The related studies trace back to 1966, when Fujita [5] first investigated the Cauchy problem of the semilinear equation

$$
\frac{\partial u}{\partial t}=\Delta u+u^{p}, \quad x \in \mathbb{R}^{n}, t>0,
$$

and showed that the problem does not have any nontrivial global nonnegative solution if $1<p<p_{c}=1+2 / n$, whereas there exist both nontrivial global (with small initial data) and non-global nonnegative (with large initial data) solutions when $p>p_{c}=1+2 / n$. Then, it was proved that $p=p_{c}$ belongs to the blow-up case by Hayakawa [10], Weissler [11], and Kobayashi [21]. We call $p_{c}$ the critical Fujita exponent and the similar results as the blowup theorem of Fujita type. There have been many various extensions of Fujita's results thence, such as different types of parabolic equations, kinds geometries of domains, different nonlinear reactions, nonhomogeneous boundaries, etc. One can refer to the survey papers $[1,2,4,8,9,12,13,17,22,23,25]$ and the references therein.

(c) The Author(s) 2019. This article is distributed under the terms of the Creative Commons Attribution 4.0 International License (http://creativecommons.org/licenses/by/4.0/), which permits unrestricted use, distribution, and reproduction in any medium, provided you give appropriate credit to the original author(s) and the source, provide a link to the Creative Commons license, and indicate if changes were made. 
The critical Fujita exponent for the Cauchy problem of

$$
\frac{\partial u}{\partial t}=\Delta u^{m}+u^{p}, \quad x \in \mathbb{R}^{n}, t>0
$$

was investigated by Galaktionov et al. in [6,7], where $p>m>1$. It was proved that $p_{c}=$ $m+2 / n$. The Cauchy problem

$$
\frac{\partial u}{\partial t}=\Delta u^{m}+|x|^{\sigma} u^{p}, \quad x \in \mathbb{R}^{n}, t>0
$$

with $\sigma>-2$ was considered by Qi [15] who proved that $p_{c}=m+(2+\sigma) / n$. The authors in [19] considered the Cauchy problem

$$
|x|^{\lambda_{1}} \frac{\partial u}{\partial t}=\Delta u^{m}+|x|^{\lambda_{2}} u^{p}, \quad x \in \mathbb{R}^{n}, t>0,
$$

where $p>m \geq 1$ and $0 \leq \lambda_{1} \leq \lambda_{2}<p\left(\lambda_{1}+1\right)-1$. It was proved that if $m<p \leq p_{c}=m+$ $\left(2+\lambda_{2}\right) /\left(n+\lambda_{1}\right)$, then every nontrivial solution of equation (4) blows up in a finite time. If $p>p_{c}$, there exist both nontrivial global and non-global solutions, with small and large $u_{0}$, respectively, to the initial-value problem (4). Martynenko and Tedeev [14] considered the Cauchy problem of the following equation with an inhomogeneous density and a source term:

$$
\rho(x) \frac{\partial u}{\partial t}=\operatorname{div}\left(u^{m-1}|\nabla u|^{q-1} \nabla u\right)+u^{p}, \quad x \in \mathbb{R}^{n}, t>0,
$$

where $q>0, m+q-2>0, p>m+q-1$, and $\rho(x)=|x|^{\lambda}$ or $\rho(x)=(|x|+1)^{\lambda}, \lambda \leq 0$. Under some restrictions on the parameters, the authors researched if any nontrivial solution to the Cauchy problem blows up in a finite time and established a sharp universal estimate of the solution near the blow-up point.

In 1991, Escobedo and Herrero [3] investigated the following coupled systems:

$$
\frac{\partial u}{\partial t}=\Delta u+v^{p}, \quad \frac{\partial v}{\partial t}=\Delta v+u^{q}, \quad x \in \mathbb{R}^{n}, t>0,
$$

where $p, q>0$, and showed that a Fujita curve is

$$
(p q)_{c}=1+\frac{2}{n} \max \{p+1, q+1\} .
$$

If $1<p q \leq(p q)_{c}$, any nonnegative nontrivial solution blows up in a finite time, whereas if $p q>(p q)_{c}$, there exist both nontrivial nonnegative global solution (with small initial data) and nonnegative blow-up solution (with large initial data). In [16], the authors studied the following Newton-filtration system:

$$
\frac{\partial u}{\partial t}=\Delta u^{m}+v^{p}, \quad \frac{\partial v}{\partial t}=\Delta v^{m}+u^{q}, \quad x \in \mathbb{R}^{n}, t>0
$$

with $0<m<1, p, q \geq 1$, and $p q>1$. It was proved that the critical Fujita exponent is

$$
(p q)_{c}=m^{2}+\frac{2}{n} \max \{p+m, q+m\} .
$$


However, for the case $m>1$, the Cauchy problem of (5) does not have any results. The main difficulty is that it has a finite velocity of the disturbance propagation, but the propagation velocities of two equations may be different, that is why we choose the two special equations.

In this paper, it is proved that the critical Fujita exponent to the problem can be formulated as $p_{c}=m+2 / n$. The main methods are inspired by [15, 19, 20, 24, 26]. We can prove that there are the blow-up solutions by using the integral estimation method. And the existence of nontrivial global solutions is shown by constructing self-similar supersolutions. For the system coupled by reactions, solutions to one equation may influence another equation. In order to guarantee the existence of the self-similar supersolutions, we set perturbation term $(|x|+1)^{\mu}$. Specifically, this ensures that the self-similar supersolutions to systems (1)-(3) of $u(x, t)=(t+1)^{-\alpha_{1}} U\left((t+1)^{-\beta_{1}}(|x|+1)\right)$ and $v(x, t)=$ $(t+1)^{-\alpha_{2}} V\left((t+1)^{-\beta_{2}}(|x|+1)\right)$ have the same support, i.e., $\beta_{1}=\beta_{2}$. The determination of $\mu$ depends on the definition of the supersolutions. By simple calculation, $\alpha_{1}=\alpha_{2}=1 /(p-1)$, $\beta_{1}=\beta_{2}=(p-m) /(2(p-1))$, and $\mu=\alpha(q-p) / \beta$. Thus, we select $(|x|+1)$ to the power of $2(q-p) /(p-m)$.

The paper is organized as follows. Section 2 lists some preliminaries such as the definition of solution, the well-posedness of problem (1)-(3). Then, several useful auxiliary lemmas are given. At last, we obtain the blow-up theorems of Fujita type for problem (1)(3).

\section{Preliminaries}

In the following, we define the subsolutions, supersolutions, as well as solutions to problem (1)-(3).

Definition 2.1 Let $0<T \leq+\infty$. A pair of nonnegative functions $(u, v)$ is called a super (sub) solution to problem (1)-(3) in $(0, T)$ if

$$
u, v \in C\left([0, T), L_{\mathrm{loc}}^{m}\left(\mathbb{R}^{n}\right)\right) \cap L_{\mathrm{loc}}^{\infty}\left(0, T ; L^{\infty}\left(\mathbb{R}^{n}\right)\right),
$$

and for any $0 \leq \varphi, \psi \in C^{2,1}\left(\mathbb{R}^{n} \times[0, T)\right)$ vanishing when $t$ near $T$ or $|x|$ being sufficiently large, the following integral identities are satisfied:

$$
\begin{aligned}
& \int_{0}^{T} \int_{\mathbb{R}^{n}} u(x, t) \frac{\partial \varphi}{\partial t}(x, t) \mathrm{d} x \mathrm{~d} t+\int_{0}^{T} \int_{\mathbb{R}^{n}} u^{m}(x, t) \Delta \varphi(x, t) \mathrm{d} x \mathrm{~d} t \\
& \quad+\int_{0}^{T} \int_{\mathbb{R}^{n}} \nu^{p}(x, t) \varphi(x, t) \mathrm{d} x \mathrm{~d} t+\int_{\mathbb{R}^{n}} u_{0}(x) \varphi(x, 0) \mathrm{d} x \leq(\geq) 0, \\
& \int_{0}^{T} \int_{\mathbb{R}^{n}} v(x, t) \frac{\partial \psi}{\partial t}(x, t) \mathrm{d} x \mathrm{~d} t+\int_{0}^{T} \int_{\mathbb{R}^{n}} \nu^{m}(x, t) \Delta \psi(x, t) \mathrm{d} x \mathrm{~d} t \\
& \quad+\int_{0}^{T} \int_{\mathbb{R}^{n}}(|x|+1)^{\mu} u^{q}(x, t) \psi(x, t) \mathrm{d} x \mathrm{~d} t+\int_{\mathbb{R}^{n}} v_{0}(x) \psi(x, 0) \mathrm{d} x \leq(\geq) 0,
\end{aligned}
$$

where $\mu=2(q-p) /(p-m)$. When it is both a supersolution and a subsolution, $(u, v)$ is called a solution to problem (1)-(3) in $(0, T)$. 
Definition 2.2 A solution $(u, v)$ to problem (1)-(3) is said to blow up in a finite time $0<$ $T<+\infty$, which is called blow-up time, if

$$
\|u(\cdot, t)\|_{L^{\infty}\left(\mathbb{R}^{n}\right)}+\|v(\cdot, t)\|_{L^{\infty}\left(\mathbb{R}^{n}\right)} \rightarrow+\infty \quad \text { as } t \rightarrow T^{-} .
$$

Otherwise, $(u, v)$ is said to be global.

Next, we will give the existence theorem and the comparison principle to problem (1)(3).

Theorem 2.1 (Local existence theorem) When $0 \leq u_{0}, v_{0} \in L_{\text {loc }}^{1}\left(\mathbb{R}^{n}\right) \cap L^{\infty}\left(\mathbb{R}^{n}\right)$, the Cauchy problem (1)-(3) admits at least one solution locally in time.

Theorem 2.2 (Comparison principle) For $t \in(0, T)$, let $\left(u^{*}, v^{*}\right)$ and $\left(u^{* *}, v^{* *}\right)$ be two solutions to system (1) and (2) with nonnegative initial data $u_{0}^{*}(x), v_{0}^{*}(x)$ and $u_{0}^{* *}(x), v_{0}^{* *}(x)$, respectively. If $\left(u_{0}^{*}(x), v_{0}^{*}(x)\right) \leq\left(u_{0}^{* *}(x), v_{0}^{* *}(x)\right)$ a.e. in $\mathbb{R}^{n}$, then $\left(u^{*}, v^{*}\right) \leq\left(u^{* *}, v^{* *}\right)$ a.e. in $\mathbb{R}^{n} \times(0, T)$.

As to the proofs of Theorems 2.1 and 2.2, one can see $[18,19]$ and the references therein.

\section{Blow-up theorems of Fujita type}

In this section, we establish the blow-up theorems of Fujita type for problem (1)-(3).

Firstly, we consider the case $p<p_{c}$.

Theorem 3.1 Assume that $m<p<p_{c}=m+2 / n$. Then any nontrivial solution to problem (1)-(3) blows up in a finite time.

Proof Let

$$
\psi(x)= \begin{cases}1, & 0 \leq|x| \leq 1 \\ \cos \frac{1}{2}(|x|-1) \pi, & 1<|x|<2 \\ 0, & |x| \geq 2\end{cases}
$$

For $l>1$, set

$$
\psi_{l}(x)=\psi\left(\frac{x}{l}\right), \quad x \in \mathbb{R}^{n}
$$

Then

$$
\left|\nabla \psi_{l}(x)\right| \leq \frac{C}{l}, \quad\left|\Delta \psi_{l}(x)\right| \leq \frac{C}{l^{2}}, \quad \frac{\left|\Delta \psi_{l}(x)\right|}{\psi_{l}} \leq \frac{C}{l^{2}}, \quad \forall x \in B_{2 l} \backslash B_{l},
$$

where $C>0$ is a constant independent of $l$, and $B_{r}$ is the ball in $\mathbb{R}^{n}$ with radius $r$ and center at the origin. Let $(u, v)$ be a nontrivial solution to problem $(1)-(3)$ in $(0,+\infty)$. Owing to Definition 2.1, we can obtain, for any $t \in(0,+\infty)$,

$$
\frac{\mathrm{d}}{\mathrm{d} t} \int_{\mathbb{R}^{n}} u(x, t) \psi_{l}(x) \mathrm{d} x \geq \int_{B_{2 l}} u^{m}(x, t) \Delta \psi_{l}(x) \mathrm{d} x+\int_{\mathbb{R}^{n}} \nu^{p}(x, t) \psi_{l}(x) \mathrm{d} x,
$$


Lens et al. Boundary Value Problems

(2019) 2019:68

Page 5 of 11

$$
\frac{\mathrm{d}}{\mathrm{d} t} \int_{\mathbb{R}^{n}} v(x, t) \psi_{l}(x) \mathrm{d} x \geq \int_{B_{2 l}} v^{m}(x, t) \Delta \psi_{l}(x) \mathrm{d} x+\int_{\mathbb{R}^{n}}(|x|+1)^{\mu} u^{q}(x, t) \psi_{l}(x) \mathrm{d} x
$$

in the distribution sense. Thus,

$$
\begin{aligned}
\frac{\mathrm{d}}{\mathrm{d} t} w_{l}(t) \geq & -\int_{B_{2 l}} u^{m}(x, t)\left|\Delta \psi_{l}(x)\right| \mathrm{d} x+l^{\theta} \int_{\mathbb{R}^{n}}(|x|+1)^{\mu} u^{q}(x, t) \psi_{l}(x) \mathrm{d} x \\
& -l^{\theta} \int_{B_{2 l}} v^{m}(x, t)\left|\Delta \psi_{l}(x)\right| \mathrm{d} x+\int_{\mathbb{R}^{n}} v^{p}(x, t) \psi_{l}(x) \mathrm{d} x,
\end{aligned}
$$

where

$$
w_{l}(t)=\int_{\mathbb{R}^{n}}\left(u(x, t)+l^{\theta} v(x, t)\right) \psi_{l}(x) \mathrm{d} x, \quad t>0
$$

and $\theta$ is to be determined. Using the Hölder inequality, one gets

$$
\begin{aligned}
\int_{B_{2 l}} & u^{m}(x, t)\left|\Delta \psi_{l}(x)\right| \mathrm{d} x \\
= & \int_{B_{2 l} \backslash B_{l}} u^{m}(x, t)\left|\Delta \psi_{l}(x)\right| \mathrm{d} x \\
\leq & \left(\int_{B_{2 l} \backslash B_{l}}(|x|+1)^{-m \mu /(q-m)}\left|\Delta \psi_{l}(x)\right|^{q /(q-m)} \psi_{l}^{-m /(q-m)}(x) \mathrm{d} x\right)^{(q-m) / q} \\
& \times\left(\int_{B_{2 l} \backslash B_{l}}(|x|+1)^{\mu} u^{q}(x, t) \psi_{l}(x) \mathrm{d} x\right)^{m / q} \\
\leq & C_{1}^{\prime} l^{n-2-(n+\mu) m / q}\left(\int_{\mathbb{R}^{n}}(|x|+1)^{\mu} u^{q}(x, t) \psi_{l}(x) \mathrm{d} x\right)^{m / q}
\end{aligned}
$$

and$$
\int_{B_{2 l}} v^{m}(x, t)\left|\Delta \psi_{l}(x)\right| \mathrm{d} x
$$$$
=\int_{B_{2 l} \backslash B_{l}} v^{m}(x, t)\left|\Delta \psi_{l}(x)\right| \mathrm{d} x
$$$$
\leq\left(\int_{B_{2 l} \backslash B_{l}}\left|\Delta \psi_{l}(x)\right|^{p /(p-m)} \psi_{l}^{-m /(p-m)}(x) \mathrm{d} x\right)^{(p-m) / p}
$$$$
\times\left(\int_{B_{2 l} \backslash B_{l}} v^{p}(x, t) \psi_{l}(x) \mathrm{d} x\right)^{m / p}
$$$$
\leq C_{1}^{\prime \prime} l^{n-2-m n / p}\left(\int_{\mathbb{R}^{n}} \nu^{p}(x, t) \psi_{l}(x) \mathrm{d} x\right)^{m / p},
$$

where $C_{1}^{\prime}, C_{1}^{\prime \prime}>0$ independent of $l$. Combining these inequalities with (6), we obtain

$$
\begin{aligned}
\frac{\mathrm{d}}{\mathrm{d} t} w_{l}(t) \geq & \left(\int_{\mathbb{R}^{n}}(|x|+1)^{\mu} u^{q}(x, t) \psi_{l}(x) \mathrm{d} x\right)^{m / q} \\
& \times\left(l^{\theta}\left(\int_{\mathbb{R}^{n}}(|x|+1)^{\mu} u^{q}(x, t) \psi_{l}(x) \mathrm{d} x\right)^{(q-m) / q}-C_{1} l^{n-2-m(n+\mu) / q}\right)
\end{aligned}
$$


Lens et al. Boundary Value Problems

(2019) 2019:68

Page 6 of 11

$$
\begin{aligned}
& +\left(\int_{\mathbb{R}^{n}} \nu^{p}(x, t) \psi_{l}(x) \mathrm{d} x\right)^{m / p} \\
& \times\left(\left(\int_{\mathbb{R}^{n}} \nu^{p}(x, t) \psi_{l}(x) \mathrm{d} x\right)^{(p-m) / p}-C_{1} l^{n-2-m n / p+\theta}\right)
\end{aligned}
$$

with $C_{1}=\max \left\{C_{1}^{\prime}, C_{1}^{\prime \prime}\right\}$. It leads to

$$
\begin{aligned}
\int_{\mathbb{R}^{n}} u(x, t) \psi_{l}(x) \mathrm{d} x \leq & \left(\int_{\mathbb{R}^{n}}(|x|+1)^{-\mu /(q-1)} \psi_{l}(x) \mathrm{d} x\right)^{(q-1) / q} \\
& \times\left(\int_{\mathbb{R}^{n}}(|x|+1)^{\mu} u^{q}(x, t) \psi_{l}(x) \mathrm{d} x\right)^{1 / q}
\end{aligned}
$$

and

$$
\int_{\mathbb{R}^{n}} v(x, t) \psi_{l}(x) \mathrm{d} x \leq\left(\int_{\mathbb{R}^{n}} \psi_{l}(x) \mathrm{d} x\right)^{(p-1) / p}\left(\int_{\mathbb{R}^{n}} \nu^{p}(x, t) \psi_{l}(x) \mathrm{d} x\right)^{1 / p}
$$

which imply that

$$
\begin{aligned}
& \int_{\mathbb{R}^{n}}(|x|+1)^{\mu} u^{q}(x, t) \psi_{l}(x) \mathrm{d} x \\
& \quad \geq \begin{cases}C_{2}^{\prime}\left(\int_{\mathbb{R}^{n}} u(x, t) \psi_{l}(x) \mathrm{d} x\right)^{q} l^{-q n+n+\mu}, & \text { if }-q n+n+\mu<0, \\
C_{3}\left(\int_{\mathbb{R}^{n}} u(x, t) \psi_{l}(x) \mathrm{d} x\right)^{q}(\ln l)^{-(q-1)}, & \text { if }-q n+n+\mu=0, \\
C_{4}\left(\int_{\mathbb{R}^{n}} u(x, t) \psi_{l}(x) \mathrm{d} x\right)^{q}, & \text { if }-q n+n+\mu>0\end{cases}
\end{aligned}
$$

and

$$
\int_{\mathbb{R}^{n}} v^{p}(x, t) \psi_{l}(x) \mathrm{d} x \geq C_{2}^{\prime \prime} l^{-n p+n}\left(\int_{\mathbb{R}^{n}} v(x, t) \psi_{l}(x) \mathrm{d} x\right)^{p},
$$

where $C_{2}^{\prime}, C_{2}^{\prime \prime}, C_{3}, C_{4}>0$ are the constants independent of $l$.

For the case $-q n+n+\mu<0$, one can select

$$
\theta=\frac{q-p}{p+1} n-\frac{\mu}{p+1}=\frac{q-p}{p+1}\left(n-\frac{2}{p-m}\right) .
$$

It follows from (7), (8), and (9) that

$$
\begin{aligned}
\frac{\mathrm{d}}{\mathrm{d} t} w_{l}(t) \geq & \left(C_{2}^{\prime} l^{-q n+n+\mu}\left(\int_{\mathbb{R}^{n}} u(x, t) \psi_{l}(x) \mathrm{d} x\right)^{q}\right)^{m / q}\left(-C_{1} l^{n-2-m(n+\mu) / q}\right. \\
& \left.+C_{2}^{\prime(q-m) / q} l^{(-q n+n+\mu)(q-m) / q+\theta}\left(\int_{\mathbb{R}^{n}} u(x, t) \psi_{l}(x) \mathrm{d} x\right)^{q-m}\right) \\
& +\left(C_{2}^{\prime \prime} l^{-n p+n}\left(\int_{\mathbb{R}^{n}} v(x, t) \psi_{l}(x) \mathrm{d} x\right)^{p}\right)^{m / p}\left(-C_{1} l^{n-2-m n / p+\theta}\right. \\
& \left.+C_{2}^{\prime \prime(p-m) / p} l^{(-n p+n)(p-m) / p}\left(\int_{\mathbb{R}^{n}} v(x, t) \psi_{l}(x) \mathrm{d} x\right)^{p-m}\right)
\end{aligned}
$$




$$
\begin{aligned}
\geq & -C l^{n-2-m n-(m-1) \theta} w_{l}^{m}(t)+C_{2} l^{-q n+n+\mu+\theta}\left(\int_{\mathbb{R}^{n}} u(x, t) \psi_{l}(x) \mathrm{d} x\right)^{q} \\
& +C_{2} l^{-n p+n-p \theta}\left(\int_{\mathbb{R}^{n}} l^{\theta} v(x, t) \psi_{l}(x) \mathrm{d} x\right)^{p},
\end{aligned}
$$

where $C_{2}=\min \left\{C_{2}^{\prime}, C_{2}^{\prime \prime}\right\}, C=\max \left\{C_{1} C_{2}^{\prime}, C_{1} C_{2}^{\prime \prime}\right\}$.

Set

$$
\Lambda=\frac{-p^{2} q n-p^{2} n+p q m n+p m n+2 p q-2 p^{2}}{(p+1)(p-m)}+n .
$$

Note that $-q n+n+\mu+\theta=-n p+n-p \theta=\Lambda$. So, for any $t>0$,

$$
\begin{aligned}
\frac{\mathrm{d}}{\mathrm{d} t} w_{l}(t) \geq & -C l^{n-2-m n-(m-1) \theta} w_{l}^{m}(t) \\
& +C_{2} l^{\Lambda}\left(\left(\int_{\mathbb{R}^{n}} u(x, t) \psi_{l}(x) \mathrm{d} x\right)^{q}+\left(\int_{\mathbb{R}^{n}} l^{\theta} v(x, t) \psi_{l}(x) \mathrm{d} x\right)^{p}\right) \\
\geq & w_{l}^{m}(t)\left(-C l^{n-2-m n-(m-1) \theta}+2^{-p} C_{2} l^{\Lambda} \cdot \min \left\{w_{l}^{p-m}(t), w_{l}^{q-m}(t)\right\}\right) .
\end{aligned}
$$

Owing to $p<p_{c}$, we get

$$
n-2-m n-(m-1) \theta<\Lambda .
$$

Recalling $w_{l}(0)$ is a nondecreasing function of $l \in(0,+\infty)$ with $\sup \left\{w_{l}(0): l \in(0,+\infty)\right\}>0$, we have

$$
C l^{n-2-m n-(m-1) \theta} \leq 2^{-(p+1)} C_{2} l^{\Lambda} \cdot \min \left\{w_{l}^{p-m}(0), w_{l}^{q-m}(0)\right\} .
$$

Then (11) and (12) yield

$$
\frac{\mathrm{d}}{\mathrm{d} t} w_{l}(t) \geq 2^{-(p+1)} C_{2} l^{\Lambda} \cdot \min \left\{w_{l}^{p}(t), w_{l}^{q}(t)\right\}, \quad t>0 .
$$

Since $q \geq p>m>1$, there exists a constant $0<T<+\infty$ such that

$$
w_{l}(t)=\int_{\mathbb{R}^{n}}\left(u(x, t)+l^{\theta} v(x, t)\right) \psi_{l}(x) \mathrm{d} x \rightarrow+\infty \quad \text { as } t \rightarrow T^{-} .
$$

Owing to supp $\psi_{l}(x)=B_{2 l}$, we find that

$$
\|u(\cdot, t)\|_{L^{\infty}\left(\mathbb{R}^{n}\right)}+\|v(\cdot, t)\|_{L^{\infty}\left(\mathbb{R}^{n}\right)} \rightarrow+\infty \quad \text { as } t \rightarrow T^{-},
$$

that is, $(u, v)$ blows up in a finite time.

For the case $-q n+n+\mu=0$, select $\theta=0$. It follows from (7), (8), and (9) that

$$
\begin{aligned}
\frac{\mathrm{d}}{\mathrm{d} t} w_{l}(t) \geq & \left(C_{3}(\ln l)^{-(q-1)}\left(\int_{\mathbb{R}^{n}} u(x, t) \psi_{l}(x) \mathrm{d} x\right)^{q}\right)^{m / q} \\
& \times\left(-C_{1} l^{n-2-m(n+\mu) / q}+C_{3}^{(q-m) / q}(\ln l)^{-(q-1)(q-m) / q}\left(\int_{\mathbb{R}^{n}} u(x, t) \psi_{l}(x) \mathrm{d} x\right)^{q-m}\right)
\end{aligned}
$$




$$
\begin{aligned}
& +\left(C_{2}^{\prime \prime} l^{-n p+n}\left(\int_{\mathbb{R}^{n}} v(x, t) \psi_{l}(x) \mathrm{d} x\right)^{p}\right)^{m / p} \\
& \times\left(-C_{1} l^{n-2-m n / p}\right. \\
& \left.+\left(C_{2}^{\prime \prime}\right)^{(p-m) / p} l^{(-n p+n)(p-m) / p}\left(\int_{\mathbb{R}^{n}} v(x, t) \psi_{l}(x) \mathrm{d} x\right)^{p-m}\right) .
\end{aligned}
$$

Owing to $n-2-m(n+\mu) / q<0, n-2-m n / p<(-n p+n)(p-m) / p$, and $w_{l}$ is a nondecreasing function of $l$, there exist $\delta^{\prime}, \delta^{\prime \prime}>0, l \gg 1$ such that

$$
\begin{aligned}
\frac{\mathrm{d}}{\mathrm{d} t} w_{l}(t) \geq & \left(C_{3}(\ln l)^{-(q-1)}\right)^{m / q}\left(\int_{\mathbb{R}^{n}} u(x, t) \psi_{l}(x) \mathrm{d} x\right)^{m} \\
& \times\left(\frac{1}{2} C_{3}^{(q-m) / q}(\ln l)^{-(q-1)(q-m) / q}\left(\int_{\mathbb{R}^{n}} u(x, t) \psi_{l}(x) \mathrm{d} x\right)^{q-m}\right) \\
& +\left(C_{2}^{\prime \prime} l^{-n p+n}\right)^{m / p}\left(\int_{\mathbb{R}^{n}} v(x, t) \psi_{l}(x) \mathrm{d} x\right)^{m} \\
& \times\left(\frac{1}{2}\left(C_{2}^{\prime \prime}\right)^{(p-m) / p} l^{(-n p+n)(p-m) / p}\left(\int_{\mathbb{R}^{n}} v(x, t) \psi_{l}(x) \mathrm{d} x\right)^{p-m}\right) \\
\geq & \delta^{\prime}\left(\int_{\mathbb{R}^{n}} u(x, t) \psi_{l}(x) \mathrm{d} x\right)^{q}+\delta^{\prime \prime}\left(\int_{\mathbb{R}^{n}} v(x, t) \psi_{l}(x) \mathrm{d} x\right)^{q} \\
\geq & 2^{-p} \delta \cdot \min \left\{w_{l}^{p}(t), w_{l}^{q}(t)\right\},
\end{aligned}
$$

where $\delta=\min \left\{\delta^{\prime}, \delta^{\prime \prime}\right\}$. Thus, there exists a constant $0<T<+\infty$ such that $(u, v)$ blows up in a finite time.

For the case $-q n+n+\mu>0$, we still select $\theta=0$. By a similar argument as the case $-q n+n+\mu=0$, we can also prove that any nontrivial solution blows up in a finite time.

Turn to the case $p>p_{c}$. To prove the existence of a nontrivial global solution to problem (1)-(3), we research self-similar supersolutions to system (1) and (2) of the following form:

$$
u(x, t)=\frac{U\left((t+1)^{-\beta}(|x|+1)\right)}{(t+1)^{\alpha}}, \quad v(x, t)=\frac{V\left((t+1)^{-\beta}(|x|+1)\right)}{(t+1)^{\alpha}}
$$

where $(x, t) \in \mathbb{R}^{n} \times[0,+\infty)$,

$$
\alpha=\frac{1}{p-1}, \quad \beta=\frac{p-m}{2(p-1)}
$$

If $U, V \in C^{1}([0,+\infty))$ with $U^{m}, V^{m} \in C^{1}([0,+\infty))$ satisfy

$$
\begin{aligned}
& \left(U^{m}\right)^{\prime \prime}(r)+\frac{n-1}{r} \cdot \frac{|x|+1}{|x|}\left(U^{m}\right)^{\prime}(r)+\beta r U^{\prime}(r)+\alpha U(r)+V^{p}(r) \leq 0, \\
& \left(V^{m}\right)^{\prime \prime}(r)+\frac{n-1}{r} \cdot \frac{|x|+1}{|x|}\left(V^{m}\right)^{\prime}(r)+\beta r V^{\prime}(r)+\alpha V(r)+r^{\mu} U^{q}(r) \leq 0
\end{aligned}
$$

for any $r>0, x \in \mathbb{R}^{n}$, then (14) implies that $(u, v)$ is a supersolution to system (1) and (2). 
Lemma 3.1 Let $p>p_{c}$ and

$$
U(r)=V(r)=\left(\eta-A r^{2}\right)_{+}^{1 /(m-1)}, \quad r \geq 0,
$$

where $s_{+}=\max \{0, s\}$ and $\eta>0$ is sufficiently small, while

$$
A=\frac{m-1}{4 m(p-1)}\left(\frac{1}{n}+\frac{p-m}{2}\right) \text {. }
$$

Then there exists sufficiently small $\eta>0$ such that $(u, v)$ given by (14) and (17) is a supersolution to the system of (1) and (2).

Proof A simple computation can lead to

$$
\begin{aligned}
& \left(U^{m}\right)^{\prime \prime}(r)+\frac{n-1}{r}\left(U^{m}\right)^{\prime}(r)+\beta r U^{\prime}(r)+\alpha U(r) \\
& \quad=\left(\frac{2 A}{m-1}\left(\frac{2 A m}{m-1}-\beta\right) r^{2} U^{1-m}(r)+\left(\alpha-\frac{2 A m n}{m-1}\right)\right) U(r), \quad 0<r<\left(\frac{\eta}{A}\right)^{1 / 2} .
\end{aligned}
$$

By the definition of $A$, we choose $\eta_{1}>0$ sufficiently small, and when $0<\eta<\eta_{1}$, we obtain

$$
\left(U^{m}\right)^{\prime \prime}(r)+\frac{n-1}{r}\left(U^{m}\right)^{\prime}(r)+\beta r U^{\prime}(r)+\alpha U(r)<0, \quad 0<r<\left(\frac{\eta}{A}\right)^{1 / 2} .
$$

Owing to $\left(U^{m}\right)^{\prime}(r)=-\frac{2 A m r}{m-1} U(r) \leq 0$, then, for $0<r<\left(\frac{\eta}{A}\right)^{1 / 2}$,

$$
\begin{aligned}
& \left(U^{m}\right)^{\prime \prime}(r)+\frac{n-1}{r} \cdot \frac{|x|+1}{|x|}\left(U^{m}\right)^{\prime}(r)+\beta r U^{\prime}(r)+\alpha U(r) \\
& =\left(U^{m}\right)^{\prime \prime}(r)+\frac{n-1}{r}\left(U^{m}\right)^{\prime}(r)+\beta r U^{\prime}(r)+\alpha U(r)+\frac{n-1}{r} \cdot \frac{1}{|x|}\left(U^{m}\right)^{\prime}(r)<0 .
\end{aligned}
$$

Similarly, for each $0<\eta<\eta_{1}$, we have

$$
\left(V^{m}\right)^{\prime \prime}(r)+\frac{n-1}{r} \cdot \frac{|x|+1}{|x|}\left(V^{m}\right)^{\prime}(r)+\beta r V^{\prime}(r)+\alpha V(r)<0,
$$

where $0<r<\left(\frac{\eta}{A}\right)^{1 / 2}$. The definitions of $U, V$ imply that

$$
r^{\mu} U^{q-1}(r) \leq A^{-\mu / 2} \eta^{[(p-1)(q-m)] /[(m-1)(p-m)]}, \quad V^{p-1}(r) \leq \eta^{(p-1) /(m-1)},
$$

where $0<r<\left(\frac{\eta}{A}\right)^{1 / 2}$. Together with (18) and (19), for sufficiently small $0<\eta_{2}<\eta_{1}$ and when $0<\eta<\eta_{2}<\eta_{1}$, we get (15) and (16). Since $U, V \in C^{1}([0,+\infty)),(u, v)$ is given by (14) and (17) which is a supersolution to system (1) and (2).

Theorem 3.2 Let $p>p_{c}$. Then there exist both nontrivial global and blow-up solutions to problem (1)-(3).

Proof The comparison principle and Lemma 3.1 yield that problem (1)-(3) with small initial data admits a nontrivial global solution. Next, let us show the existence of a blowup solution to problem (1)-(3) with large initial data. 
Fix $l>0$ and let $(u, v)$ be the solution to problem (1)-(3). Set

$$
\tilde{w}_{l}(t)=\int_{\mathbb{R}^{n}}(u(x, t)+v(x, t)) \psi_{l}(x) \mathrm{d} x, \quad t \geq 0 .
$$

From the first half of the proof of Theorem 3.1, we know that when $-q n+n+\mu<0$, we use the Hölder inequality to get

$$
\begin{aligned}
\frac{\mathrm{d}}{\mathrm{d} t} \tilde{w}_{l}(t) & \\
\geq & -C_{1} l^{n-2-m n}\left(\left(\int_{\mathbb{R}^{n}} u(x, t) \psi_{l}(x) \mathrm{d} x\right)^{m}+\left(\int_{\mathbb{R}^{n}} v(x, t) \psi_{l}(x) \mathrm{d} x\right)^{m}\right) \\
& +\int_{\mathbb{R}^{n}}(|x|+1)^{\mu} u^{q}(x, t) \psi_{l}(x) \mathrm{d} x+\int_{\mathbb{R}^{n}} \nu^{p}(x, t) \psi_{l}(x) \mathrm{d} x \\
\geq & -C_{1} l^{n-2-m n} \tilde{w}_{l}^{m}(t)+C_{2}^{\prime} l^{-q n+n+\mu}\left(\int_{\mathbb{R}^{n}} u(x, t) \psi_{l}(x) \mathrm{d} x\right)^{q} \\
& +C_{2}^{\prime \prime} l^{-n p+n}\left(\int_{\mathbb{R}^{n}} v(x, t) \psi_{l}(x) \mathrm{d} x\right)^{p} \\
\geq & \tilde{w}_{l}^{m}(t)\left(-C_{1} l^{n-2-m n}+2^{-p} C_{2} \cdot \min \left\{\tilde{w}_{l}^{p-m}(t), \tilde{w}_{l}^{q-m}(t)\right\}\right), \quad t \geq 0,
\end{aligned}
$$

where $C_{2}=\min \left\{C_{2}^{\prime} l^{-n p+n}, C_{2}^{\prime \prime} l^{-q n+n+\mu}\right\}$ is a positive constant that depends only on $n, p, q$, and $l$. If $\left(u_{0}, v_{0}\right)$ is so large that

$$
C_{1} l^{n-2-m n} \leq 2^{-(p+1)} C_{2} \cdot \min \left\{\tilde{w}_{l}^{p-m}(0), \tilde{w}_{l}^{q-m}(0)\right\},
$$

then (20) leads to

$$
\frac{\mathrm{d}}{\mathrm{d} t} \tilde{w}_{l}(t) \geq 2^{-(p+1)} C_{2} \cdot \min \left\{\tilde{w}_{l}^{p}(t), \tilde{w}_{l}^{q}(t)\right\}, \quad t>0 .
$$

By the same method above, one can show that $(u, v)$ must blow up in a finite time. Similarly, we can prove that blow-up solutions to problem (1)-(3) with large initial data exist when $-q n+n+\mu \geq 0$.

\section{Acknowledgements}

The authors would like to thank the referees for their valuable comments and suggestions which improved the original manuscript.

Funding

This work is supported by the National Natural Science Foundation of China (Grant Nos. 11571137, 11601182, and 11801211), by the Department of Science and Technology of Jilin Province (20180520213JH), and by the Education Department of Jilin Province (JJKH20180114KJ).

Availability of data and materials

Not applicable.

Competing interests

The authors declare that they have no competing interests. 


\section{Publisher's Note}

Springer Nature remains neutral with regard to jurisdictional claims in published maps and institutional affiliations.

Received: 21 November 2018 Accepted: 31 March 2019 Published online: 05 April 2019

\section{References}

1. Andreucci, D., Cirmi, G., Leonardi, S., Tedeev, A.: Large time behavior of solutions to the Neumann problem for a quasilinear second order degenerate parabolic equation in domains with noncompact boundary. J. Differ. Equ. 174, 253-288 (2001)

2. Deng, K., Levine, H.: The role of critical exponents in blow-up theorems: the sequel. J. Math. Anal. Appl. 243, 85-126 (2000)

3. Escobedo, M., Herrero, M.: Boundedness and blow up for a semilinear reaction-diffusion system. J. Differ. Equ. 89, 176-202 (1991)

4. Fira, M., Kawohl, B.: Large time behavior of solutions to a quasilinear parabolic equation with a nonlinear boundary condition. Adv. Math. Sci. Appl. 11, 113-126 (2001)

5. Fujita, H.: On the blowing up of solutions of the Cauchy problem for $u_{t}=\Delta u+u^{1+\alpha}$. J. Fac. Sci., Univ. Tokyo, Sect. I 13, 109-124 (1966)

6. Galaktionov, V:: Blow-up for quasilinear heat equations with critical Fujita's exponents. Proc. R. Soc. Edinb. A 124, 517-525 (1994)

7. Galaktionov, V., Kurdjumov, S., Mikhailov, A., Samarskii, A.: On unbounded solutions of the Cauchy problem for the parabolic equation $\partial u / \partial t=\nabla(u \sigma \nabla u)+u^{\beta}$. Dokl. Akad. Nauk SSSR 252, 1362-1364 (1980)

8. Guo, W., Lei, M.: Critical Fujita curves for a coupled reaction-convection-diffusion system with singular coefficients. J. Jilin Univ. Sci. 54, 183-188 (2016)

9. Guo, W., Wang, X., Zhou, M.: Asymptotic behavior of solutions to a class of semilinear parabolic equations. Bound. Value Probl. 2016, 68 (2016)

10. Hayakawa, K.: On nonexistence of global solutions of some semilinear parabolic equations. Proc. Jpn. Acad. 49, 503-525 (1973)

11. Kobayashi, K., Siaro, T., Tanaka, H.: On the blowing up problem for semilinear heat equations. J. Math. Soc. Jpn. 29, 407-424 (1977)

12. Levine, H., Zhang, Q.: The critical Fujita number for a semilinear heat equation in exterior domains with homogeneous Neumann boundary values. Proc. R. Soc. Edinb., Sect. A 130(3), 591-602 (2000)

13. Li, H., Wang, X., Nie, Y., He, H.: Asymptotic behavior of solutions to a degenerate quasilinear parabolic equation with a gradient term. Electron. J. Differ. Equ. 2015, 295 (2015)

14. Martynenko, A., Tedeev, A.: Cauchy problem for a quasilinear parabolic equation with a source term and an inhomogeneous density. Comput. Math. Math. Phys. 47(2), 238-248 (2007)

15. Qi, Y.: The critical exponents of parabolic equations and blow-up in $\mathbb{R}^{n}$. Proc. R. Soc. Edinb., Sect. A 128(1), 123-136 (1998)

16. Qi, Y., Levine, H.: The critical exponent of degenerate parabolic systems. Z. Angew. Math. Phys. 44, 249-265 (1993)

17. Qi, Y., Wang, M.: Critical exponents of quasilinear parabolic equations. J. Math. Anal. Appl. 267, 264-280 (2002)

18. Quittner, P., Souplet, P.: Superlinear Parabolic Problems. Blow-up, Global Existence and Steady States. Birkhäuser Advanced Texts: Basler Lehrbücher. Birkhäuser, Basel (2007)

19. Wang, C., Zheng, S.: Critical Fujita exponents of degenerate and singular parabolic equations. Proc. R. Soc. Edinb., Sect. A 136(2), 415-430 (2006)

20. Wang, C., Zheng, S., Wang, Z:: Critical Fujita exponents of a class of quasilinear equations with homogeneous Neumann boundary data. Nonlinearity 20, 1343-1359 (2007)

21. Weissler, F:: Existence and non-existence of global solutions for semilinear equation. Isr. J. Math. 6(1), 85-126 (2000)

22. Winkler, M.: A critical exponent in a degenerate parabolic equation. Math. Methods Appl. Sci. 25, 911-925 (2002)

23. Wu, Z., Zhao, J., Yin, J., Li, H.: Nonlinear Diffusion Equations. World Scientific, River Edge (2001)

24. Zheng, S., Wang, C.: Large time behaviour of solutions to a class of quasilinear parabolic equations with convection terms. Nonlinearity $21(9), 2179-2200$ (2008)

25. Zhou, M., Li, H., Guo, W., Zhou, X.: Critical Fujita exponents to a class of non-Newtonian filtration equations with fast diffusion. Bound. Value Probl. 2016, 146 (2016)

26. Zhou, Q., Nie, Y., Han, X.: Large time behavior of solutions to semilinear parabolic equations with gradient. J. Dyn. Control Syst. 22(1), 191-205 (2016)

\section{Submit your manuscript to a SpringerOpen ${ }^{\circ}$ journal and benefit from:}

- Convenient online submission

- Rigorous peer review

- Open access: articles freely available online

- High visibility within the field

- Retaining the copyright to your article

Submit your next manuscript at $\boldsymbol{~ s p r i n g e r o p e n . c o m ~}$ 